\title{
Les fantômes de Prague
}

\section{Gérard-Georges Lemaire}

\section{(2) OpenEdition}

Journals

Édition électronique

URL : https://journals.openedition.org/rbnu/3208

DOI : $10.4000 /$ rbnu.3208

ISSN : 2679-6104

\section{Éditeur}

Bibliothèque nationale et universitaire de Strasbourg

\section{Édition imprimée}

Date de publication : 1 novembre 2011

Pagination : 54-67

ISSN : 2109-2761

\section{Référence électronique}

Gérard-Georges Lemaire, «Les fantômes de Prague », La Revue de la BNU [En ligne], 4 | 2011, mis en ligne le 01 novembre 2011, consulté le 09 août 2021. URL : http://journals.openedition.org/rbnu/3208 ; DOI : https://doi.org/10.4000/rbnu.3208

\section{(c) (i) (3)(2)}

La Revue de la BNU est mise à disposition selon les termes de la Licence Creative Commons Attribution - Pas d'Utilisation Commerciale - Partage dans les Mêmes Conditions 4.0 International. 


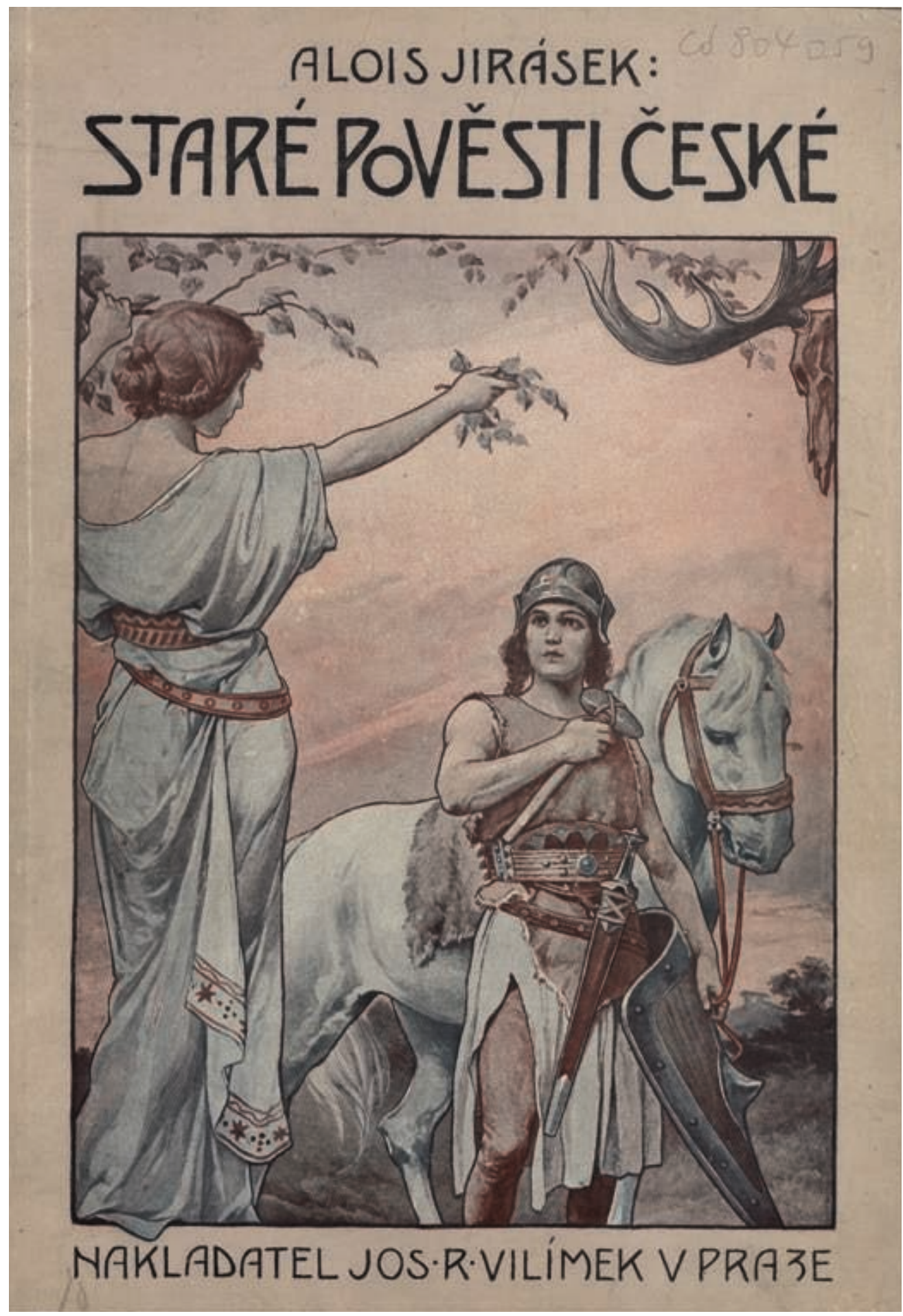

Couverture des Antiques légendes tchèques d’Alois Jirásek (Prague, 1917 ; coll. BNU) 


\section{LES FANTÔMES DE PRAGUE}

\section{Mise en bouche}

$\mathrm{A}$

ngelo Maria Ripellino l'a rappelé dans cette somme inégalable qu'est Praga magica ${ }^{1}$ : la capitale de la Bohême est l'une des trois villes formant le triangle magique en Europe. Il constaste que " la littérature pragoise nous ensevelit sous un florilèges de noires habitations, lieux maléfiques par excellence ; Leppin dit de Severin que lorsqu'il regardait à travers ses paupières mi-closes, les maisons de Prague devenaient fantastiques : "Etait-ce la faute de cette ville avec ses façades obscures, le silence de ses grandes places, son ardeur morte ? Il avait toujours l'impression que des miroirs invisibles l'effleuraient $"{ }^{2}$.

Dans la seconde moitié du $19^{\mathrm{e}}$ siècle, alors que la langue tchèque avait failli sombrer corps et bien après des siècles de domination habsbourgeoise, des écrivains de talent avaient remis au goût du jour la vieille Prague avec ses us et coutumes et sa singularité - je pense en premier lieu aux Contes de Malá Strana ${ }^{3}$ de Jan Neruda (1834-1891), mais surtout aux innombrables légendes qui ont été exhumées peu à peu depuis cette période. Deux auteurs de talent - mais ils n'ont pas été les seuls - se sont employés non seulement à les retrouver, à les dépoussiérer et à les recueillir dans des anthologies, mais aussi à leur donner une véritable forme littéraire : c'est l'oeuvre accomplie par Alois Jirásek (1851-1930) et par Frantisek Langer (1888-1965). Le premier d'entre eux, dans ses Antiques légendes tchèques $(1894)^{4}$, a consigné l'essentiel des grands mythes populaires de la Bohême, qui renforçaient la particularité culturelle de l'identité de ce vaste terri- toire aux mille et un châteaux, mais aussi l'idée que l'esprit qui y régnait y était ancré depuis des temps reculés et constituait l'héritage commun de tous les Tchèques. C'est sans nul doute la conséquence du sursaut nationaliste qui avait conduit à certains excès : un écrivain avait voulu faire passer pour authentiques des incunables qu'il avait fabriqués de sa main dans la langue ancienne ("glova "), qui ne remontait qu'au premier millénaire de notre ère et était longtemps demeurée un avatar du protoslave. Cristina Bongiorno décrit Jirásek comme l'un des plus importants protagonistes de la renaissance de cette culture condamnée par l'Empire autrichien, tombée en désuétude depuis le début du $17^{e}$ siècle et qui s'était réaffirmée sous le règne de François-Joseph $\mathrm{I}^{\mathrm{er}}$. Son roman, Tête de chien, en témoignait amplement. Ce fils de boulanger, qui se reconnaissait dans l'esprit rebelle de la réforme de Jan Hus, mais qui défendait des positions réactionnaires, contribua avec succès à la prise de conscience historique du peuple tchèque et à son droit à l'indépendance.

L'histoire de l'ancien royaume de Bohême et celle de Prague étaient marquées par une suite continuelle d'événements désastreux en plus des maux frappant le reste de l'Europe, de la peste noire à la guerre de Trente Ans. La célèbre bataille de la Montagne Blanche (1620) en fut l'expression dramatique : les forces protestantes du roi Frédéric V (surnommé le Roi Hiver à cause de la brièveté de son règne) furent vaincues par la ligue catholique. Les têtes des vingtsept chefs protestants furent exposées longtemps sur le pont Charles. La Bohême perdit son indépendance pendant plusieurs siècles et les réformés durent fuir. 
L'Eglise triomphait et modela le pays et encore plus Prague selon sa volonté en imposant l'esthétique de la démesure architecturale et stylistique du baroque. La culture tchèque périclita d'autant plus que cette longue guerre avait fait des ravages irréparables au point de rendre les campagnes quasiment désertiques. Parmi toutes les nouvelles fantastiques et pittoresques qu'on peut lire dans les ouvrages de ces deux auteurs, quelques-unes, et non des moindres, évoquent le monde étrange et très inquiétant des esprits. Dans le cas de Jirásek, l'apparition de la Dame blanche en est sans doute la démonstration la plus saisissante. Cette nouvelle nous fait remonter le cours

du temps jusqu'à la Renaissance (il

$\mathrm{y}$ est question d'un seigneur qui

était le bras droit de Charles

IV). Ce fantôme d'une femme

très belle est devenu illustre

parce qu'il hantait plusieurs

familles, et donc des lieux

très différents : " En un

moment quelconque son

ombre errait dans les châteaux de Tele, de Bechyn, de Krumlov, de Hradek et de Trebon. Alors, dans ces endroits, advenaient avec

une fréquence particulière des

événements tristes ou heureux :

naissances, morts ou noces somp-

tueuses. Apparaissait toujours vêtue

de blanc une femme austère, mais atti-

rante, la tête recouverte d'un voile de veuve. On disait qu'elle ne portait des gants de couleur noire qu'à l'occasion des funérailles ${ }^{5}$. Elle pouvait surgir tout d'un coup dans l'encadrement d'une fenêtre ou au sommet d'une tour, passer d'une pièce à l'autre, toujours vêtue tout de blanc, même la nuit. Ce fantôme immaculé n'inspirait pas la peur. Il pouvait même se montrer secourable, par exemple lors de la naissance du fils du seigneur Jost de Rozenberk de Krumlov en 1539 : " La femme prit dans ses bras le nouveau-né qui s'endormit aussitôt. Elle s'occupa de cet enfant tous les soirs. Mais la nourrice la surprit et lui arracha l'enfant des mains avec violence. Ce bambin grandit et on lui raconta cette mystérieuse présence protectrice qui accompagna ses premiers jours. Il s'appelait Petr Vok. Il décida un jour de faire abattre le mur précisément là où la Dame blanche apparaissait : en fait, elle lui avait indiqué l'endroit où était scellé un antique trésor de la famille. Elle se présenta aussi aux yeux du vieux seigneur Jachym en 1604 dans le château de Hradek. Une lueur éblouissante éclaira la salle et elle le guida jusqu'à une chapelle où se trouvait déjà prête l'huile sainte que le prêtre devait utiliser pour lui administrer l'extrême-onction. Et elle se montra encore un jeudi saint pour annoncer que la fête du printemps aurait désormais lieu ce même jour. Les fidèles sont venus plus tard la vénérer dans la cuisine où l'on préparait le repas des pauvres chaque année et aussi dans la petite chapelle où sa trace était demeurée. C'était toujours l'amour qui la poussait en dehors de sa sépulture et l'incitait à errer sur les terres de ses nobles ancêtres pour prendre soin de leur progéniture ou pour prévenir ses parents des joies et des douleurs futures, leur enseignant comment ils devaient naviguer sur le fleuve de la vie, leur révélant enfin quand leur heure avait sonné et qu'ils allaient bientôt rejoindre leurs pères $\aleph^{6}$.

La Prague du temps jadis passait pour être peuplée de fantômes de toutes sortes. Dans Tristes lieux, l'écrivain remémore la date fatidique du 21 juin 1621 quand, au début de l'Ere des Ténèbres ("Temno "), on conduisit au gibet les chevaliers qui avaient conduit leurs troupes contre les armées habsbourgeoises et celles de leurs alliés sur la Bilá Hora, la Montagne Blanche, le 8 novembre 1620. Depuis, ils apparaissaient la veille de leur exécution, un jour qui marquait la fin de la dynastie des rois de Bohême. La nuit, défilaient les ombres des nobles et des citoyens qui avaient été exécutés : "Ils se rassemblaient tous là où avait eu lieu le supplice et paisiblement, à pas lents, ils traversaient la place, entraient dans l'église de Tyn, s'agenouillaient devant l'autel et, après avoir reçu le corps du Seigneur sous les deux formes du pain et du vin, se dissolvaient dans le néant ${ }^{7}$. Frantisek Langer, le frère de Jiri, l'ami hassidim de Franz Kafka, médecin de formation, un des habitués du café Union et du café Arco, fiefs de l'avant-garde pragoise, où il fit la connaissance de Karel Capek dont 


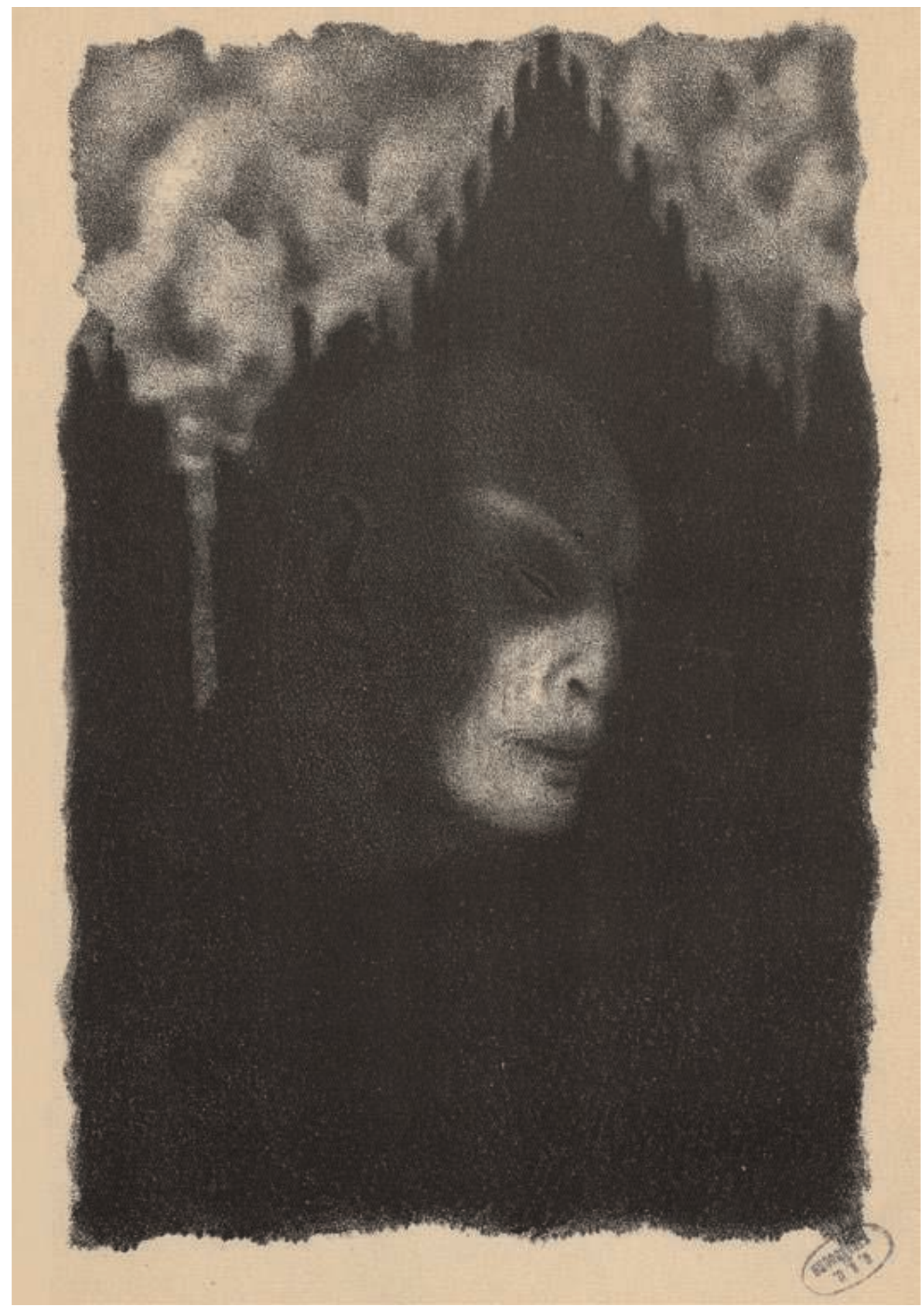

Frontispice de l'édition originale du Golem de Gustav Meyrink

(Leipzig, Kurt Wolff, 1915 ; coll. BNU) 


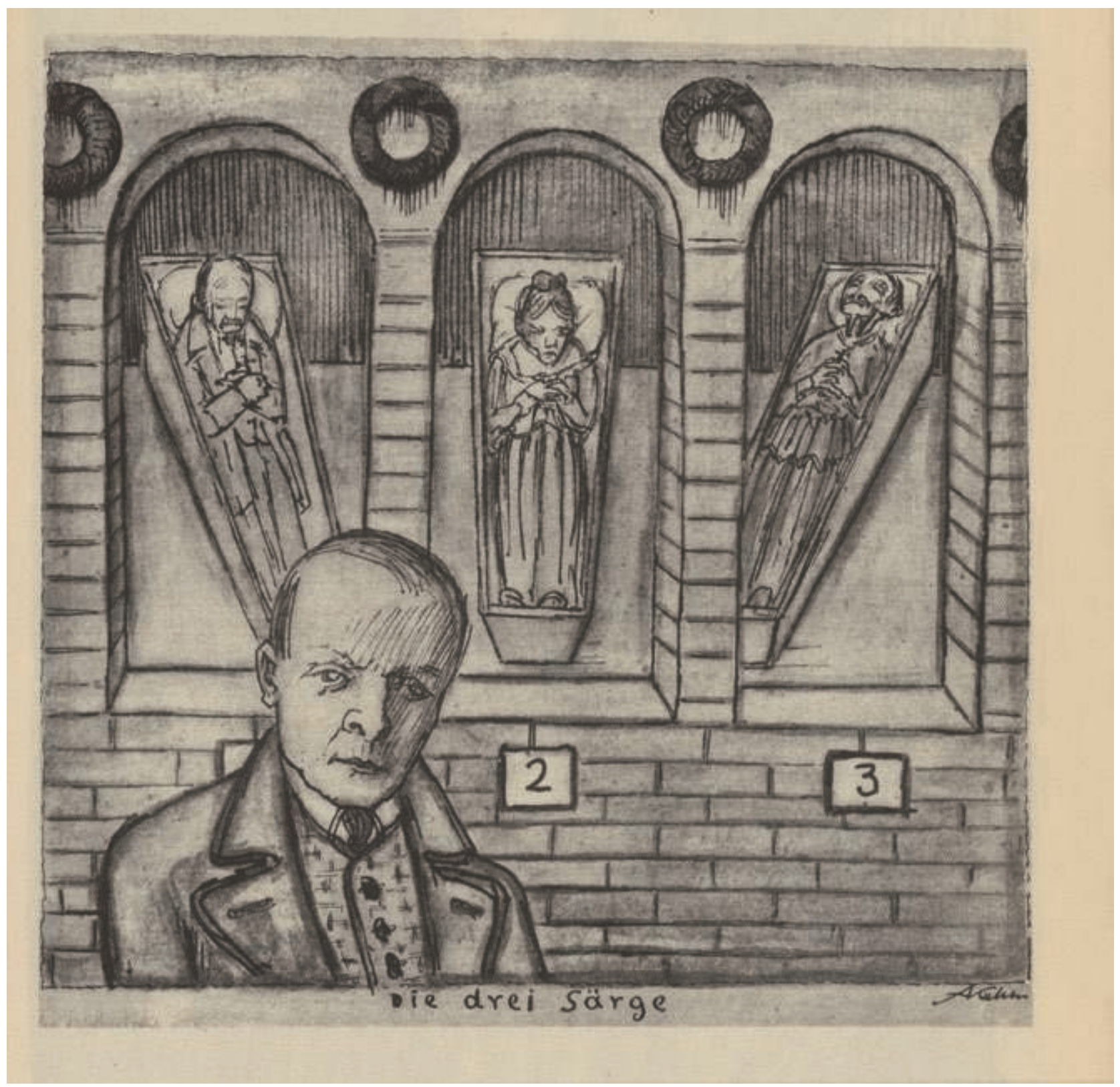

Autoportrait d'Alfred Kubin,

extrait de l'ouvrage Dämonen und Nachtgesichte

(Dresde, Carl Reissner, 1926),

intitulé « Autoportrait devant la salle mortuaire » (coll. BNU) 
il a subi l'influence dans la perspective de son réalisme très spécifique, a été l'un des représentants de la jeune littérature tchèque d'après la Grande Guerre. Bien que son œuvre soit essentiellement théâtrale, il s'est attaché à faire revivre les plus significatives des innombrables légendes qui ont accompagné l'histoire tourmentée de Prague. Dans ses Légendes pragoises (1956), il a, comme Jirásek, réuni des histoires noires de l'époque où les fantômes avaient droit de cité dans ses demeures et ses ruelles.

Quand on lit sa nouvelle intitulée Le Templier sans tête, on assiste à la curieuse destinée d'un des chevaliers de l'ordre militaire des Templiers. Le grand maître, en gravissant les marches le conduisant à sa mort, jura qu'il reviendrait pour que la vérité puisse éclater aux yeux de tous. Il vécut depuis lors dans un étage surélevé de la maison sise au $n^{\circ} 6$ de la Templorá ulice où se trouvait autrefois le siège de l'ordre. Les habitants de cette maison n'étaient pas gênés par sa présence. Vers onze heures du soir, le Templier décapité descendait au rez-de-chaussée, sortait, montait à cheval et parcourait les rues en semant l'effroi. Il faisait toujours le même parcours derrière l'église de Tyn. Il était vêtu d'une merveilleuse armure d'acier et, sur ses épaules, flottait la cape blanche frappée de la croix rouge et, à ses flancs, se trouvait une épée d'acier de Damas. Au fil des ans, il commença à avoir soif. Il avisa une taverne et il y entra. Il tira sa tête du heaume. Et il but toute la bière. Celle-ci se répandit sur le sol. Le tavernier lui conseilla de placer sa tête sur son cou. Pour le remercier, il offrit à ce dernier et à sa fille un anneau avec une pierre précieuse. L'heureux bénéficiaire de ce don fit reconstruire son local et l'appela U Zlathéro prstenu (A l'anneau d'or) et il existe toujours. Les siècles ayant passé, on a abattu les maisons du quartier pour édifier des immeubles de briques. Les rues aussi disparurent. Pour le Templier, ces démolitions ne pouvaient être accomplies que par des ennemis. Il s'en prit à des militaires. Et, un beau soir, le templier ne retrouva plus sa tête...

Dans une autre nouvelle de ce beau recueil, Maya Magica, force est de constater que la culture pragoise, et pas seulement son histoire malheureuse plus ou moins magnifiée, est placée à l'enseigne du fantastique pur, du mystérieux, parfois même du sordide. Comme si tout ce qui les concernait était lié aux forces du mal, à la déchéance physique et morale et au malheur. Fort heureusement, toutes les légendes rassemblées dans les Prazske legendy de Langer ne sont pas sombres et tragiques. Il relate des contes charmants dont les personnages sont des figures bienveillantes du folklore, de petits génies des eaux par exemple comme " le Vodnik, le petit homme des eaux, que le peuple nomme aussi hastrman et bestrman, déformations amusantes de l'allemand Wassermann. Cette créature vivant dans les cours d'eau, dans les gouffres, dans les lacs, dans les étangs, n'est pas inconnue dans d'autres terres slaves, mais elle n'a jamais eu autant de relief que dans ces régions bohémiennes et moraves ${ }^{8}$. Son aspect est drolatique, à en croire Ripellino, puisque son corps de nain est vert et qu'il porte des pantalons rouges. En dehors de sa taille et de sa couleur de peau, son aspect est étonnant : ses cheveux sont aussi verts que sa peau et sont recouverts de plantes aquatiques alors que ses yeux sont ronds comme des soucoupes. Il explique : " Le peintre Arcimboldo en aurait peutêtre fait le portrait comme un croisement allégorique de rhizopodes, d'ichtyoïdes, de crustacés et d'autres larves lacustres $" 9$.

Langer raconte les faits et gestes de plusieurs de ces petits génies des eaux bien intentionnés comme celui de la grève de Vyserad ou bien celui du pont Charles près de Malá Strana, sous la quatrième arche, monsieur Josef. Il s'était assigné la bonne garde des noyés emportés par le courant, comme par exemple de « quelques-unes des très belles âmes de 1848 qui défendirent la barricade de la Vieille Ville et se sacrifièrent pour la terre natale et pour sa liberté $\aleph^{10}$. Ce protecteurs des humains morts dans les flots de la Vlatva peut aussi sauver un suicidé, comme ce fut le cas pour une jeune fille qui voulut se jeter à l'eau tandis qu'il fumait tranquillement.

I

En somme, Prague ne manque ni de fantômes, ni de revenants, ni d'esprits divers et variés. La mémoire collective qui la constitue est particulièrement féconde en la matière. Dans sa littérature, dans son art aussi, surtout avant l'avènement des avant-gardes historiques (mais Langer et d'autres poursuivent cette tradition), c'est un univers spectral qui vient à l'esprit pour la définir, plus que de simples affaires d'apparitions à proprement parler. Le Golem de Gustav Meyrink (1868-1832), cet Autrichien catholique, mais amateur de sciences occultes et membre de nombreuses sociétés plus ou moins secrètes, est sans conteste le 


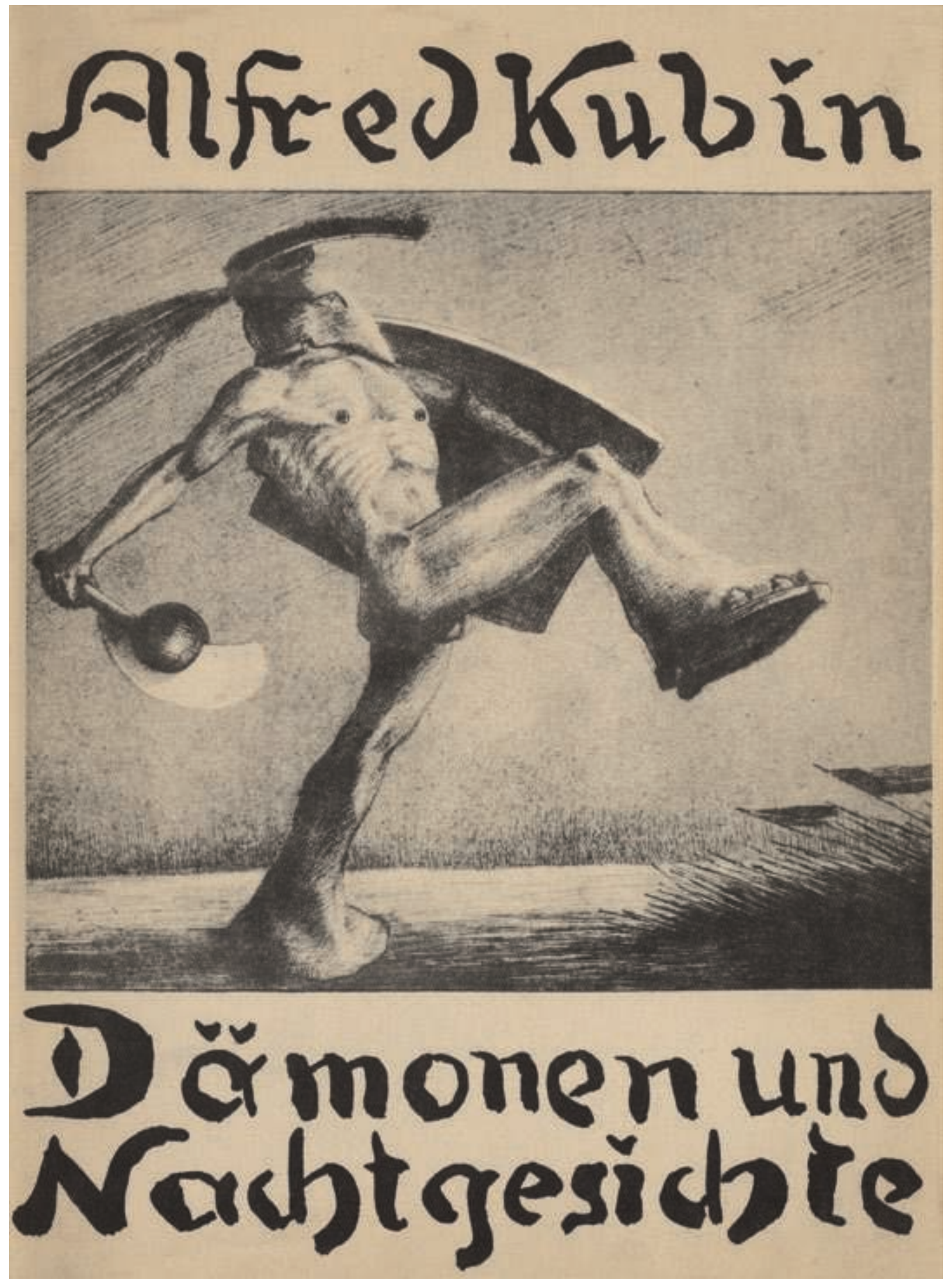

Frontispice de Dämonen und Nachtgesichte d'Alfred Kubin, reprenant un de ses dessins les plus célèbres,

La guerre, réalisé vers 1901-1902

(Dresde, Carl Reissner, 1926 ; coll. BNU) 
livre qui symbolise le mieux ce goût prononcé pour l'étrange et le fantastique. Lorsqu'il vivait à Prague, il avait un petit cercle d'admirateurs fervents qui se réunissaient autour de lui au café Continental et toute personne étrangère à cette coterie était assez mal venue de la déranger quand le maître parlait à voix presque basse à ces hommes qu'il fascinait ${ }^{11}$. L'histoire du Golem, ce monstre d'argile façonné par le rabbin Löw (qui vécut à l'époque du fantasque empereur Rodolphe II de Habsbourg qui avait choisi Prague comme capitale) pour défendre la communauté hébraïque des attaques brutales qu'elle subissait, avait déjà fait l'objet d'innombrables versions orales ou écrites, les unes sérieuses, les autres de pure

fantaisie $^{12}$. Patrizia Runfola nous in-

dique qu'Aloïs Jirásek en a donné sa

propre version dans ses Anciennes

légendes tchèques (avec l'Histoire

du grand rabbin) $)^{13}$. C'était le

mythe par excellence qui avait germé et mûri au sein du vieux ghetto juif, qui finit par disparaître sous la pioche des démolisseurs à l'époque où naissait Kafka. Meyrink a sans doute connu ses derniers vestiges. Qu'il ne fût pas Juif est ici d'une importance cruciale pour comprendre de quelle façon il a interprété cette figure terrible, intimement liée à l'histoire et à la religion d'un peuple et d'un quartier de la ville formant une totalité en soi. Mais ce monde-là, aussi petit fût-il, eut une influence énorme sur l'ensemble de la population pragoise et surtout sur ses lettrés. De toute façon, à en croire Meyrink dans ce livre, c'était Prague tout entière qui se révélait menaçante avec " cette vie sournoise, hostile, qui rayonne d'elles [ses vieilles demeures] $\aleph^{14}$. Et le narrateur y exprime le sentiment angoissé qu'il avait de Prague, qui semblait dépositaire de secrets terrifiants, de présences menaçantes et de cauchemars sans nom : "Depuis une génération que j'habite ici, l'impression s'est ancrée en moi, indestructible, qu'il y a des heures de la nuit et de l'aube à peine grisonnantes, où elles tiennent un mystérieux conseil muet. Souvent un faible tremblement que l'on ne saurait expliquer traverse alors les murs, des murmures courent sur leurs toits, tombent dans les gouttières - et nous les percevons distinctement, les sens enrouillés, sans chercher leur origine. Souvent j'ai rêvé que j'épiais leur manège spectral, apprenant ainsi avec une stupeur angoissée que ces maisons étaient les vraies maîtresses de la rue, capables de manifester leur vie et leurs sentiments, puis de les enfouir à nouveau en elles - les dissimulant à ceux qui habitent là pour les faire ressurgir à la tombée de la nuit, avec un intérêt usuraire $\aleph^{15}$.

A partir de ces méditations sur la nature double et perverse de cette ville, il généralise alors le principe qui a donné vie au monstre de glaise animé par un seul mot : les habitants de Prague perdraient leur âme si l'on introduisait dans leur cerveau " n'importe quel microscopique concept... $»^{16}$. Il relate ensuite l'histoire du Golem et celle de son illustre créateur, appelé à la cour de l'empereur " pour évoquer les esprits et les faire apparaître ${ }^{17}$. C'est un vieillard qui raconte cette légende du monstre destructeur à Prokop et Penath. Il leur dit qu'il est impossible de le décrire. Celui-ci ressurgit dans les ruelles et les impasses tous les trente-trois ans. Ce phénomène inexplicable ne provoque aucun affolement dans la population. "Chaque fois ", poursuit le vieil homme, " un homme totalement inconnu, imberbe, le visage jaunâtre et de type mongol, se dirige à travers le quartier juif vers la rue de la Vieille Ecole d'un pas égal, curieusement trébuchant, comme s'il allait tomber en avant d'un instant à l'autre, puis soudain disparaît. En général il tourne un angle de rue et se volatilise. Une autre fois, on dit qu'il a décrit un cercle pour revenir à son point de départ : une très vieille maison dans le voisinage de la synagogue $\aleph^{18}$. Et le narrateur prétend même qu'il l'a rencontré et qu'il en est resté profondément marqué, comme au fer rouge. Il suppose qu'il " est peut-être constamment au milieu de nous sans que nous nous en apercevions ${ }^{19}$.

En réalité, plus qu'une nouvelle conception du thème $\mathrm{du}$ Golem, ce roman est la traduction d'une quête ésotérique plus vaste dans un univers livré au plus étrange et au plus inquiétant. Il est ici une sorte de 
deus ex machina. Dans le chapitre intitulé "Spectre", Penrath, au terme d'une course folle dans les souterrains de Prague, voit prendre forme et volume la figure d'une carte à jouer, celle du Fou. Il se rend compte qu'il ne s'agit là que du double de lui-même. Il parvient à maîtriser cette apparition troublante et la ligote. Puis, soulagé, il la voit se dissoudre dans le néant : « Et tandis qu'il devenait de plus en plus petit et se recroquevillait de nouveau dans sa carte à jouer, je me suis levé, je suis allé à lui et je l'ai mis dans ma poche, le Fou ${ }^{20}$. Mais quand il sortit dans les rues de Prague, les personnes qu'il croisait prenaient peur et se mettaient à le poursuivre : il comprit alors que le Golem, c'était lui, et nul autre!

Patrizia Runfola dépeint avec sagacité ce nouveau Golem comme une sorte de menace indistincte, mais pourtant omniprésente : "Ainsi la fantaisie collective donnait-elle naissance à la réalité, mais l'ombre du doute demeurait, imperceptible : peut-être la position astrologique des étoiles sous lesquelles il avait été créé par la puissance de l'esprit du rabbin se répétant régulièrement, le fantôme se déplaçait-il vraiment dans les ruelles lugubres et sales du ghetto, poussé par l'angoisse qui le tourmentait de retrouver la vie matérielle, et vivait-il de nouveau dans l'imagination des âmes-succubes dans la hantise d'une mésaventure imminente, dont la menace pesait d'une manière toujours plus effrayante $"^{21}$. Pour Angelo Maria Ripellino, c'est " l'indice d'une épidémie spirituelle "22. A ses yeux, ce n'est plus alors un mannequin d'argile, " mais une apparence fuyante, énigmatique, nébuleuse, un 'Spuk' (revenant) ${ }^{23}$. Il met d'ailleurs l'accent sur cette propension générale, dans la tradition et la culture pragoises, à imaginer la ville comme le refuge privilégié des trépassés demeurés cachés là pour rappeler aux citadins des événements épouvantables issus de leur passé. Il nous renvoie à la fiction pléthorique de Francis Marion Crawford (1854-1909), The Witch of Prague (1891), qui est une sorte de compendium très arrangé de ces macabres fantaisies s'attachant à telle ou telle bâtisse médiévale ou de la Renaissance. Par exemple, il parle d'un estaminet frappé par une malédiction séculaire : "Au Puits d’or, orné sur sa façade de saints en stuc noir qui protègent de la peste, fut un temps une citadelle de spectres ${ }^{24}$. Il évoque les autres auteurs qui ont abordé ces sujets fantastiques, comme Josef Svátek (1835-1897), l'auteur des Mystères de Prague (1868) : dans le château de Bonneval sis à Malá Strana, la nuit venue, un squelette se lamente et dans le palais
Pachta, dans la Celetná ulice, se trouvait " une arche de spectres et d'esprits ". Il parle aussi de l'œuvre de Popelka Biliánová (1862-1941). Ces auteurs ont exploré cette veine insondable et inépuisable et Ripellino en tire cette conclusion : "La littérature pragoise nous ensevelit sous un florilège de noires habitations ensorcelées, lieux maléfiques par excellence $"{ }^{25}$. Le Golem est la manifestation la plus formidable de ce climat surnaturel qui s'est imposé dans la capitale tchèque.

\section{II}

Alfred Kubin (1877-1959), originaire de Leitmeritz en Bohême du nord, a connu une enfance tourmentée. Son équilibre nerveux rendit cette période de sa vie très difficile. La mort de sa mère, alors qu'il n'avait que dix ans, le marqua à jamais, tout autant que l'affliction de son père. Quand il va étudier à Salzbourg, il éprouve une fascination irrésistible pour les cadavres. Alors qu'il fait son apprentissage dans l'atelier photographique de son oncle par alliance à Klagenfurt, il ressent le désir de se suicider sur la tombe de sa mère ; mais le révolver qu'il avait dérobé chez son parent s'est enrayé. Son oncle se fâche et le renvoie. Après cette mésaventure, il veut s'engager dans l'armée. Mais sa candidature est rejetée. Il insiste, supplie, et le conseil de révision cède malgré son jeune âge. On l'envoie à Laibach, capitale de la Carniole. Lors des préparatifs de la cérémonie funèbre du commandant de division, il a un comportement si étrange qu'on décide de le réformer. Il s'est évanoui en entendant la marche funèbre et est saisi d'une sorte de délire. Il est envoyé à l'hôpital psychiatrique de l'armée à Graz où il reste un mois. De retour chez son père, il connaît encore des périodes de convulsions inexplicables. C'est alors qu'un ami de la famille s'est aperçu de ses dons pour le dessin et l'on s'accorde pour le faire inscrire à l'Académie des beaux-arts de Munich. Il a des phases de tremblements aigus qui l'empêchent de dessiner. Mais il parvient néanmoins à progresser dans cet art. C'est alors qu'en lisant Emmanuel Kant il éprouve une sensation pour le moins curieuse : " j'eus la sensation d'être une entité pour laquelle mon corps, la pièce où j'étais, les expériences cubistes qui m'occupaient alors ne formaient plus qu'un tout confus ${ }^{26}$. Et elle s'est répétée par la suite. Il se fiance à l'âge de vingtcinq ans mais sa promise, après être venue lui rendre visite, meurt au bout de dix jours. Il décrit ce qu'il 


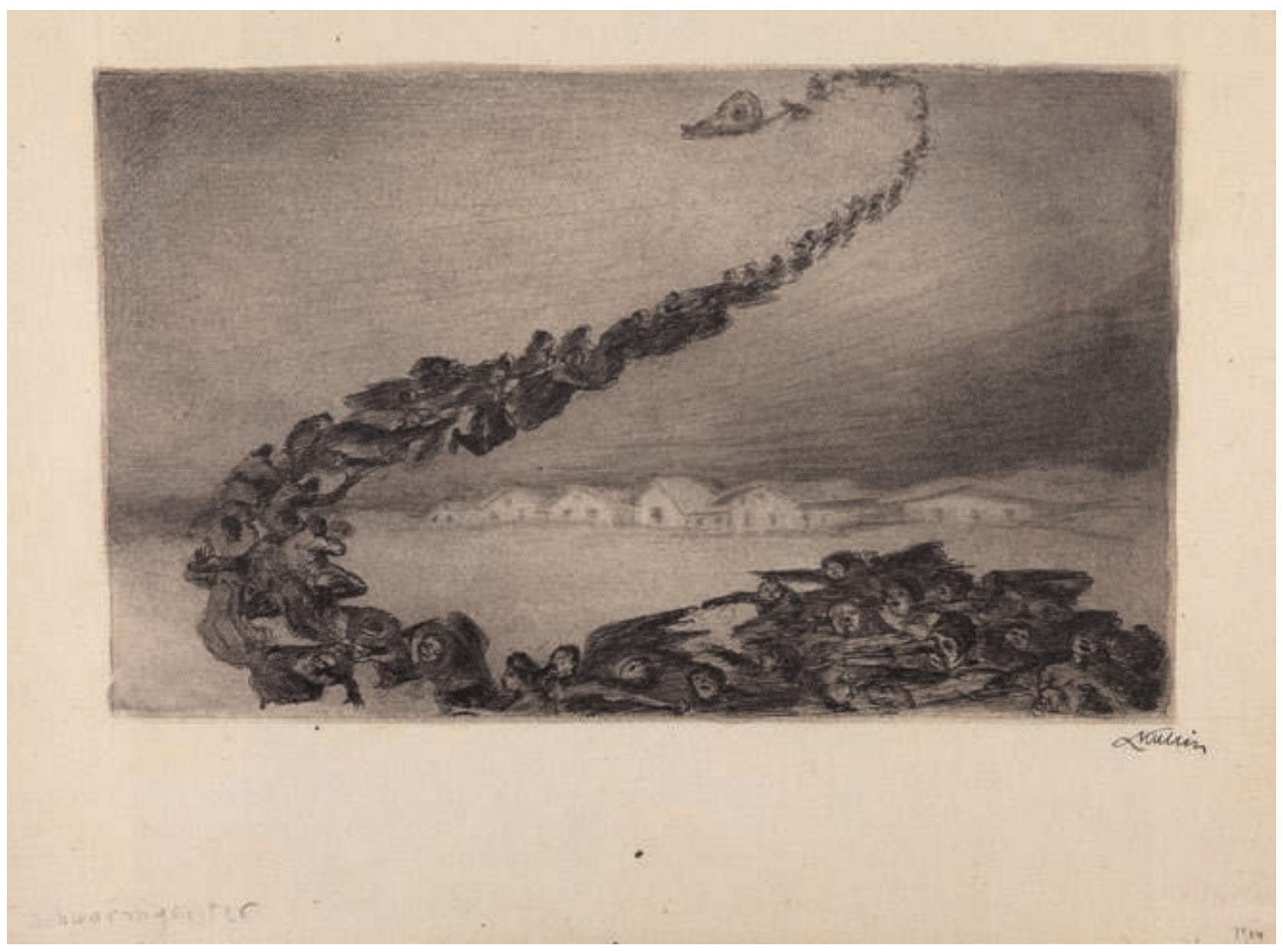

Les esprits de la foule (Schwarmgeister),

dessin, autour de 1903 


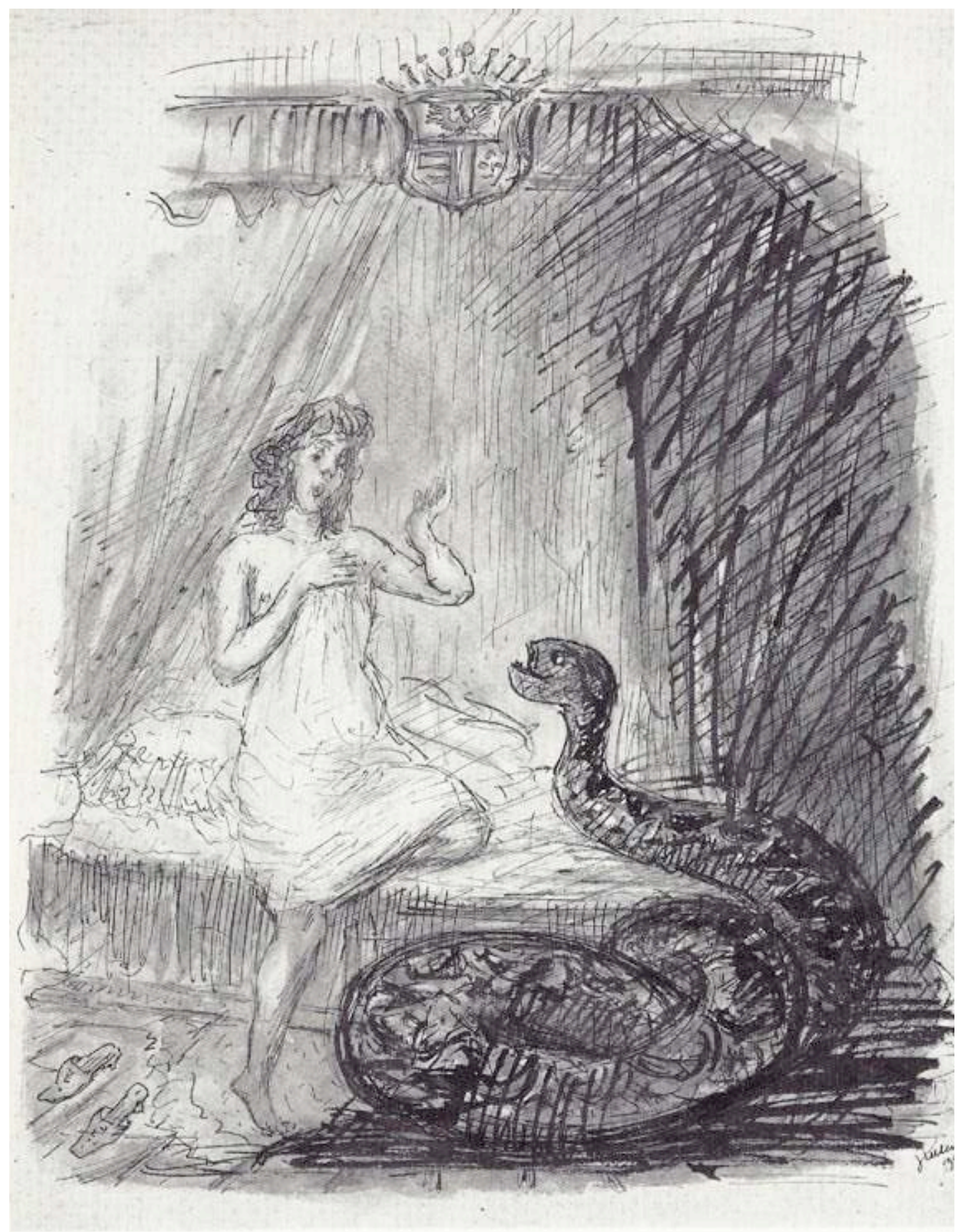

Serpent, dessin, vers 1939 
traverse après ce nouveau drame : " Extravagances et excès de toutes sortes se succédèrent dans un désordre échevelé... " ${ }^{27}$. Par bonheur, il fait la connaissance d'une autre jeune fille qui lui plaît et l'épouse en 1904. Il semble trouver un certain équilibre. Peu après, le couple s'installe à Zwickledt, en Haute-Autriche.

Quelques années passent et, en 1908, après avoir fait évoluer considérablement son œuvre graphique, il écrit " en douze semaines à peine ${ }^{28}$ un long roman qu'il baptise Die andere Seite (L'autre côté). Ce que Kubin ne raconte pas dans ses mémoires (il ne le fera que dans un court essai autobiographique), c'est qu'une bonne partie des dessins qu'il a exécutés pour illustrer sa fiction, qui est la représentation fantasmagorique de Prague, placée sous un éclairage encore une fois nocif et occulte, étaient destinés à un autre ouvrage, celui de Gustav Meyrink, Le Golem. Ce dernier a pu vivre ses rêves les plus fous dans cette cité qui convenait à ses penchants cabalistiques avant de devoir la quitter en 1904 lorsqu'il est accusé d'avoir fait des malversations dans son établissement bancaire. C'est en Bavière qu'il commence à rédiger ce livre. En janvier 1907, il prend contact avec Kubin, qui séjourne à Munich, pour lui demander d'illustrer son roman. Il lui annonce ensuite qu'il va lui envoyer les premiers chapitres - ce qu'il a fait, au moins pendant quelques mois. Mais Kubin ne voit pas arriver les suivants et cela l'exaspère : "Cette règle en soi absurde ", souligne-t-il en 1933 dans Comment j'illustre mes livres, elle " n'a pu être respectée que pour les premiers chapitres [...] Impatient, j'ai atten$\mathrm{du}$ en vain la suite du manuscrit dont je dépendais matériellement et $[. .$.$] je n'ai pas arrêté de changer de$ manière de dessiner. Je ne pouvais pas attendre la fin du Golem " ${ }^{29}$. Meyrink, en panne d'inspiration, ne lui envoie plus rien. C'est ainsi que de son propre aveu, " [il a] utilisé les dessins qu'[il avait] déjà réalisés pour [son] propre roman, L'autre côté ${ }^{30}$. Après la mort de son père, qui le bouleverse et l'ébranle nerveusement, il se remet au dessin avec beaucoup de bonheur et de réussite. C'est alors qu'il a l'idée subite de son propre roman, fruit de ses crises esthétiques et personnelles. Il a paru en $1909^{31}$. Le livre connaît un grand succès et ne cessera plus d'être réédité.

Bon nombre de connaisseurs de l'œuvre de Kubin ont perçu le parallélisme biaisé entre cet ouvrage et celui de Meyrink, qui ne paraît qu'en 1915 et qui se vend en plein conflit mondial à cent mille exemplaires. Ce qui était Prague de manière explicite chez l'un est changé chez le second en une cité imaginaire, Perla.
Mais Kubin s'est presque complètement débarrassé de l'histoire du Golem (toutefois, à la fin du livre, le tyran Patera devient un géant destructeur) et surtout de toutes ses implications hébraïques, historiques et ésotériques et de ses innombrables versions littéraires, de Josef Svátek à Vrchilicky et au poète allemand Detlev von Liliencron (1844-1909) qui lui a consacré une ballade en 1898 (Der Golem), sans parler des commentaires savants de doctes rabbins, qui ont fleuri à la fin du $19^{\mathrm{e}}$ et au début du $20^{\mathrm{e}}$ siècle (au début en hébreu, ensuite en allemand), comme Der Golem Prag de Chaïm Block, qui a inspiré le cinéaste Paul Wegener qui l'a adapté en 1914.

Ripellino a fait une réflexion très pertinente sur le passage de l'atmosphère du roman de Gustav Meyrink à celui d'Alfred Kubin : "Au lieu de transférer les Asiatiques à Prague, il déplaça Prague [...] vers le cœur de l'Asie, au-delà de Samarkand $\aleph^{32}$. Ce n'est pas le seul déplacement effectué : Kubin n'a retenu de Prague que son essence et l'a libérée de son passé si lourd. Ce qui fait la différence fondamentale entre les deux œuvres littéraires, c'est justement la vision de Prague qu'elles véhiculent chacune à sa façon : c'est pour Kubin un monde d'invention qui appartient de bout en bout au rêve et à son empire, où le soleil ne brille jamais. Mais le narrateur de cette histoire qui prend le train pour se rendre dans une région très lointaine nous apprend : " En gros, c'était pareil à l'Europe centrale, mais c'était aussi différent ${ }^{33}$. On y retrouve néanmoins le célèbre Cinquième quartier, le ghetto ancestral, tel qu'il figure dans le Golem en dépit de nuances significatives : "Cette petite partie de la ville, avec ses quatre mille habitants, des Latins, des Slaves et des Juifs, passait pour un quartier mal famé. Une foule bigarrée s'y entasse en vrac, dans de vieilles maisons de bois. Avec ses ruelles borgnes et ses coupe-gorges nauséabonds, ce quartier ne faisait pas particulièrement l'orgueil de Perla $»^{34}$.

Que Kubin ait désiré faire de Prague la capitale de l'" empire des rêves" (Traumreich) est le point décisif de son aventure d'écrivain. Ce puissant "Traumreich " est l'expression du monde fabuleux et souvent déconcertant, plein d'incongruités et de bizarreries risibles ou pénibles, chargé d'anxiété et de peur, qu'il avait créé de dessin en dessin. Si les êtres de l'ombre et des nuits cauchemardesques de Meyrink sont remplacés par des êtres appartenant aux récits extravagants et débridés du rêve, ils sont engendrés par une transposition déformée et truquée de leur univers d'ori- 
gine, et donc révélateurs de la réalité, mais aussi des mythes et des légendes : ainsi l'Américain qui arrive à Perla et y fait un long discours où il exhorte ses habitants en ces termes : " Fantômes déraisonnables, vous n'êtes vraiment plus bons à rien ; vos moelles sont vouées au diable, à la duperie la bribe d'intelligence qui vous reste... $"{ }^{35}$. A ce stade précis de l'histoire, les citoyens de ce royaume commencent à rencontrer leurs sosies chemin faisant : " En effet, dès leur arrivée, les nouveaux se trouvèrent en face de leurs sosies : cela déclencha toutes sortes d'agaceries et de contrariétés car non seulement beaucoup de rêveurs nouveaux venus ressemblaient par la physionomie et par l'allure à certains fixés de longue date, mais il semblait encore qu'ils avaient eu, dans leur façon de s'habiller, l'intention de copier l'original $\star^{36}$. Le narrateur fait à son tour des rencontres qui le troublent profondément : " je rencontrai une dame qui ressemblait à ma défunte femme, comme un œil ressemble à l'autre ${ }^{37}$. Il découvre enfin que c'est en fait l'épouse d'un facteur d'orgues de la cour. Il en tire aussitôt les conséquences : "Il me semblait que j'étais fou " $3^{8}$. La destruction inéluctable et tragique de Perla dans une atmosphère de fin des temps apocalyptique renforce cette impression que la ville est à la fois antique et enracinée dans l'histoire présente, métamorphosée certes, en proie à une guerre féroce, sorte de prémonition de la chute de l'immense empire des Habsbourg craquant déjà de partout. A la fin de l'ouvrage, le voyageur a une révélation qui l'étonne : " D'un seul coup, je reconnus tout, c'était le hameau où j'avais passé mon enfance ${ }^{39}$. Cela n'empêche pas que l'espace l'environnant continue à se dilater et que le temps s'étire lui aussi dans un sens ou dans l'autre. Patera, le régent implacable de cette métropole improbable est devenu subitement un géant qui se met à détruire tout sur son passage, aidé par l'Américain, et tout finit en catastrophe. Après la mort de Patera, Perla n'est plus qu'un champ de ruines dont les troupes européennes triomphantes s'emparent.

Notre narrateur retrouve le monde " normal " et se réveille dans une maison de santé. Il pense dans son lit de convalescent : " Au regard de l'empire des rêves, la réalité me faisait l'effet d'une odieuse caricature ${ }^{40}$. Peu à peu, il reprend conscience de l'originelle dualité $\mathrm{du}$ monde et il se risque " de nouveau dans la vie " ${ }^{41}$, en sachant bien que Dieu est sans cesse aux prises avec un antagoniste formidable. Il a surmonté la fascination de la mort et il a la faculté désormais de péné- trer dans les contradictions inhérentes à l'humanité banale.

\section{En guise de conclusion}

Ainsi, cette Prague qu'il connaît au fond si peu et où il a connu Franz Kafka, qu'il a influencé de manière indélébile pour la rédaction du Procès ${ }^{42}$, est vouée dans son œuvre de fiction à une fin funeste. Ses habitants y sont à la fois des spectres imaginaires et la traduction dans le langage des rêves de ce qui s'est avéré sa vérité dans ses pensées et dans ses songes. Elle a condensé en elle toutes ses obsessions et toutes ses hantises. Patrizia Runfola le raconte avec une grande justesse dans Prague au temps de Kafka : " un premier amour semblait alléger l'atmosphère oppressante qui régnait en maîtresse dans la vie de Kubin, quand la noire mégère revint au galop, la faux levée pour emporter son unique affection. Dès lors, les cauchemars et obsessions morbides ne le quittèrent plus et envahirent ses dessins comme une armée frénétique et maléfique : il dessinait des femmes enceintes à la difformité révoltante, souvenir de ses premiers jeux sensuels, à l'âge de onze ans avec une femme enceinte - prédilection qui continuait à le hanter et qui manifestait son mépris absolu pour le genre masculin. Son esprit se mit alors à cultiver une sensibilité malsaine pour la violence de la nature : catastrophes, orages, incendies, torrents en crue. Les plus noires manifestations de la violence s'auréolaient d'une lumière séduisante : rixes, arrestations, scènes sanguinaires au marché aux bestiaux, le spectacle répugnant des pièces de viandes découpées sur le marbre par des couteaux aiguisés. Ces résurgences continuelles et nauséabondes de l'abjection engendrèrent en fin de compte chez le jeune Kubin une aversion sourde pour la vie [...] Une nuit, alors qu'il cherchait à apaiser l'angoisse qui lui serrait la gorge, il entra dans un théâtre de variétés. Il y eut la révélation du monde grotesque, de la bestialité humaine, des figures difformes, physiquement et moralement, que son imagination engendrait dans un tourbillon vertigineux et infatigable d'images sarcastiques, gémissantes, du bestiaire humain [...] Certes, il savait bien que l'imagination, en imprimant un sceau brûlant sur sa vie, dominait despotiquement ses humeurs, et que la fatigue physique, en même temps qu'une sensibilité accrue à la douleur, reviendraient tourmenter sans trêve son âme $»^{42}$.

Kubin a fini par exorciser les démons de ce peuple 
d'épouvante qui l'a habité si longtemps et qui l'a enfin laissé en paix. Die andere Seite a opéré une catharsis et les fantômes dont il a consigné avec délectation autant qu'avec effroi les apparences sur le papier ne sont plus maintenant que les protagonistes apprivoisés de ses fantasmes qui, peu à peu, l'ont quitté. C'est là que s'est joué dans la dimension romanesque et dans celle de la création artistique ce qui s'est joué en son for intérieur, le rêve prenant une autre consistance, n'étant plus le signe annonciateur des événements à venir comme dans les religions de l'Antiquité, ni la manifestation de Satan, ni le signe d'une maladie mentale, mais un révélateur dont les clefs ésotériques donnent accès à l'indicible du vivant, donnant matière à interprétation afin d'avoir accès à l'autre côté de la conscience.

Die andere Seite a vraiment fait passer Kubin de l'autre côté de lui-même, cette fois du côté de la lumière, du côté de la paix intérieure. Lui qui aimait tant Goya, Klinger, von Stuck ou Rops n'a pas renoncé à son univers fertile en monstres et démons. Mais il n'en est plus la victime. Il a écrit dans son autobiographie : " L'écrire m'a fait prendre conscience que les plus hautes valeurs ne se trouvent pas seulement dans les moments bizarres, grandioses ou comiques de l'existence, mais que les choses pénibles, les choses indifférentes, les choses quotidiennes et accessoires renferment les mêmes secrets " ${ }^{43}$. Les fantômes ne sont plus que des signes qu'il trace au crayon et à l'encre. Et le rêve a changé de nature : il s'est fait son allié pour sous-tendre sa quête artistique.

\section{Gérard-Georges Lemaire}

\footnotetext{
Notes

1 - Praga magica, Angelo Maria Ripellino, traduit de l'italien par Jacques Michaut-Paternò, " Terre humaine ", Plon, 1993

2 - Praga magica, op. cit. p. 238

3 - Les contes de Malá Strana, Jan Neruda, traduit du tchèque par François Kérel, Artia, Prague, 1963

4 - Racconti e leggende della Praga d'Oro, Alois Jirásek, traduit du tchèque et présenté par Cristina Bongiorno, "Oscar Mondadori ", Arnaldo Mondadori editore, Milan, 1989

5 - Ibidem, p. 161

6 - Ibid., p. 168

7 - Ibid., p. 116
}

8 - Préface d'Angelo Maria Ripellino, L'uomo delle acque, pour Leggende praghesi, Frantisek Langer, traduit du tchèque par Giuseppe Dierna, edizioni E/O, Rome, 1981 ; publié à l'origine dans La Fiera letteraria, 1952, VI, $n^{\circ} 41$, p. 4

9 - Ibidem, p. 5

10 - Leggende praghesi, op. cit., p. 30

11-Cf. Les cafés littéraires, Gérard-Georges Lemaire, éditions de la Différence, 1997

12- Cf. Le Golem, Moshe Idel, traduit de l'anglais par Cyrille Aslanoff, préfacé par Henri Atlan, "Patrimoines/judaïsme ", Cerf, 2007

13- Prague au temps de Kafka, Patrizia Runfola, traduit de l'italien et préfacé par Gérard-Georges Lemaire, "Les essais ", éditions de la Différence, 2002

14- Le Golem, Gustav Meyrink, traduit de l'allemand par Denise Meunier, Stock, 1969, p. 26

15- Ibidem

16- Ibid., p. 27

17- Ibid., p. 41

18 -Ibid., p. 42

19-Ibid., p. 44

20-Ibid., p. 94

21- Prague au temps de Kafka, op. cit., p. 118

22- Praga magica, op. cit., p. 202

23- Ibidem

24- Cité in Praga magica, op. cit., p. 237

25- Ibidem, p. 238

26- Ma vie, Alfred Kubin, présenté et traduit de l'allemand par Christophe David, éditions Allia, 2000, pp. 48-49

27- Ibidem, p. 51

28 - Ibid., p. 66

29- Cité in Kafka et Kubin, Gérard-Georges Lemaire, "Les essais ", éditions de la Différence, 2002, p. 94

30-Le travail du dessinateur, Alfred Kubin, traduit de l'allemand par Christophe David, Allia, 1999, p. 113

31-Chez Georg Müller, Leipzig/Munich

32-Praga magica, op. cit., p. 230

33-L'autre côté, Alfred Kubin, traduit de l'allemand par Robert Valançay, " 391 ", Eric Losfield, 1962, p. 48

34-Ibidem

35-Ibid., pp. 152-153

36-Ibid., p. 153

37-Ibid.

38-Ibid.

39-Ibid., p. 245

40-Ibid., p. 263

41 - Ibid.

42-Prague au temps de Kafka, op. cit., pp. 125-128

43-Ma vie, op. cit., p. 67 